\title{
Archetypes of Enterprise Social Network Users
}

\author{
Christian Oettl, Thomas Berger, Markus Böhm, Manuel Wiesche, Helmut Krcmar \\ Technical University of Munich \\ \{c.oettl, thomas.berger\}@tum.de, \{markus.boehm, manuel.wiesche, krcmar\}@in.tum.de
}

\begin{abstract}
Investments in enterprise social networks (ESNs) have increased rapidly in recent years. However, an ESN utilization intensity develops slowly, and there are a few well-grounded approaches to understand ESN usage. To elaborate on different archetypes of ESN users, we conducted a case study that comprised 28 interviews with a large IT services company. We present a model to characterize ESN users and classify them as archetypes based on the following two dimensions: individual openness to ESNs and perceived task-fit. We determine six archetypes of ESN users, namely, power users, limited users, reluctant users, repudiators, hidden champions, and question marks. From a theoretical viewpoint, this study contributes to the discussion around user typology of ESN users and the utilization intensity, acceptance, and value contribution of ESNs. In practice, results provide an orientation to organizations that intend to address both ESN users and the organization to increase the utilization intensity of ESNs.
\end{abstract}

\section{Introduction}

Enterprise social networks (ESNs) receive increasing attention as well as fast dissemination, especially in large organizations [8], [15]. Specifically, ESNs are internal web-based user centered social platforms that allow their users to communicate with colleagues; to identify potential communication partners; to create, publish, or edit their own content; and to access content created by other users [1], [15]. Thus, ESN users are encouraged to actively and openly contribute (e.g., to discussions or by voicing criticism) with a "sharing is caring" attitude.

Personalized user profiles characterize ESNs as social platforms that link content and authors [8]. Previous research already identified positive effects of ESN usage. In particular, large multinational organizations will promote the introduction and development of ESNs in the future [8].

However, even if ESNs are implemented in organizations, their value contribution appears unclear because $80 \%$ of projects do not fulfill expectations [16]. The typical ROI of any social technology becomes positive when $15 \%$ to $25 \%$ of employees use such technology extensively and companies should not assume that "If we build it, they will come" [3].

This was also observed in the company that was considered in this study. In 2014, a large multinational IT service provider started a radical metamorphosis from an email centered to an ESN centered organization. The implemented solution provides typical key functionalities that characterize an ESN as follows: individual personal profiles, online work communities and collaboration spaces, activity feeds, feedback functionality, instant messaging, tagging, global content sharing, analytical options to measure trends and community health, and similarity with established internet social networks. However, ESN utilization intensity develops slowly, and the value contribution of an ESN is perceived as ambiguous.

Based on this unique case, we investigate "what are the archetypical users of ESN systems," "how users value the ESN system they use," and "what kind of obstacles do the users perceive" to increase understanding of reasons for the actual system use of ESN users. We believe that there is a lack of research on the archetypes of ESNs that includes the description, identification, and structuring of ESN users. This is a significant step to understand actual use and actual value contribution of ESNs.

This study is organized as follows: first, we introduce existing research and the theoretical background of this study. Second, we describe the research method as well as the process of data collection and analysis. In the results section, we depict a model of identified archetypes of ESN users that specially focuses on their perceived added values and obstacles. We proceed by discussing results, theory, and implications of the study before providing a conclusion. 


\section{Theoretical background}

This study presents a model that characterizes ESN users and classifies them into archetypes based on the following two dimensions: individual openness to ESNs and perceived task-fit.

The model is based on (1) literature on ESNs (usage; effects through ESNs; management, leadership and governance for ESNs; value contribution and performance measurement; cultural aspects; architecture and design; theories, research design, and methods) [24], (2) literature relevant for user typification, and (3) existing technology acceptance theories and models.

Benefits of ESNs include that messages from others are visible to users (message transparency) and that the structure of their communication networks is explained (network translucence) [14]. Previous studies already indicated improved communication across hierarchical and organizational boundaries [7], [36], improved knowledge transfer and expert search [2], [9], [14], [17], enhanced innovational strength [11], [14], [18] as well as the establishment and strengthening of social ties [12], [27] as added values of ESNs. Although it is obvious that knowledge workers should be interested in optimal access to knowledge to maximize productivity, the specified benefits are perceived differently by individuals [36].

As a summary, the focus of studies moves "from identifying benefits towards ways of quantifying benefits" of ESNs [34]. Although literature relevant for user typification exists, to the best of the authors' knowledge, there is no approach that combines individual and productivity related dimensions. The diffusion of innovations (DOI) literature [23] distinguish users by how quickly they adopt new technologies, and user typification mentioned by Velasquez et al. [28] and Jahnke [13] is based on a user's role within an online community. They state that it is not feasible to expect that all individuals use a system with equal frequency. This when combined with Orlikowski [21] who demonstrated that different roles also lead to conflicts of interest and incentives it seems understandable that users do not use a system with similar frequency. In contrast, this is not in line with the premise that knowledge workers are generally interested in the best possible access to knowledge to raise their productivity. Third, participation inequality mentioned in topical literature on social networks, online communities, and discussion forums [22], [20] indicates that a small minority of users perform most of the work and identifies contributors and consumers.

However, none of the aforementioned literature on user typification combines individual and task related dimensions and neither is particularly aimed at ESN users. It is necessary to consider, existing models and theories of IS acceptance to craft a model that characterizes users of ESNs.

Influencing factors of user adoption are intensively discussed within existing technology acceptance theories and models [25]. The most prevalent and recognized models include (1) the "task-technology fit" (TTF) model that proposes a theoretical model that focuses on task characteristics and technology characteristics (2) the "technology acceptance model" (TAM) and its advancements (TAM2; TAM3) that center on independent variables including perceived usefulness and perceived ease of use, and (3) the "unified theory of acceptance and use of technology" (UTAUT) that focuses on the expected future impact (e.g., performance expectancy and effort expectancy) of IS usage [6], [10], [29], [32].

\section{Research method}

In this section, we present the research design as well as the process with which the developed model of archetypes of ESN users is crafted. This study comprises of a purely exploratory research design of a single case study with 28 participants that follow the guidelines listed by Yin [35].

We selected this company as it changed their internal communication system from an email centered towards an ESN centered approach in a radical manner. Specifically, the company introduced its ESN as strategically important while it was voluntary in use and fitted perfectly to synthesize (1) trait related and (2) task and outcome related drivers for ESN use.

We retrieved the data from 28 expert interviews from more than 10000 potential ESN users ranging from $16 \mathrm{~min}$ to $40 \mathrm{~min}$ between May 2015 and July 2015. We selected participants in consultation with three representatives of the partner company (ESN project lead, roll-out manager, and ambassador) and determined the following premises: (1) participants hold different business roles that are typical for industrial companies (human resources, engineering, leadership, business support) to mitigate a potential deviation of the results based on the specifics of an IT company; (2) participants are selected well-balanced in terms of their age, sex, and an estimate of their attitude towards ESNs from the experience of the partner company; and (3) participants are approached by ambassadors to convey voluntariness of participation and openness for feedback.

We designed the interview guide with two major premises: (1) task and outcome related as well as individual trait related constructs from existing user acceptance models are addressed via open questions, 
and (2) the "individual reaction to using IT" is part of the basic concept that underlies all user acceptance models (a) functions as the chronological starting point for the study and (b) defocuses constructs from existing body of knowledge related to an initial intention to use (prior to the ESN roll out).

Although extant studies offer a well acknowledged toolbox of existing user acceptance models, we decided to not fully apply those as we were interested in the detailed explanations and developments of ESN usage. Therefore, we determine that it is reasonable to explore the social aspects of ESN use by asking open questions to subsequently match the explored constructs with existing models. Second, we aim to keep the model simple to focus on task and outcome related as well as individual trait related constructs.

The interview guide covers questions on the following topics: (1) demographics, business role, (mandate description and requirements) and communication style of interviewees; (2) use of ESNs (active/passive; task-related/non-task-related); (3) perceived added values and problems/obstacles of ESNs; (4) personal attitude towards social networks in general and especially towards ESNs; and (5) perceived future potential of ESNs and expected impact on interviewees.

We analyzed the data in the following three steps: First, we applied the structuring content analysis [19] to synthesize relevant constructs from the interview data. Second, we matched the identified constructs (a) to task and outcome as well as (b) individual trait relatedness, and (c) to constructs of existing user acceptance models, to analyze and understand differences to constructs of existing user acceptance models. Third, we coded the interviews.

In order to determine task-fit we coded: (a) ESN usage, frequency, and relative importance, (b) perceived task and outcome related added value and obstacles. In order to determine openness to ESNs we coded: (a) attitude towards ESNs, (b) perceived ability to use ESNs, (c) promotion of and resistance against ESNs, (d) perceived future potential/importance of ESNs, and (e) perceived environmental influence (as shown in Table 1).

Furthermore, we classified the identified variables for each interviewee based on the data as low, medium, and high. Subsequently, we classified the subjects in our model with the two dimensions perceived task fit and individual openness to ESNs. Finally, we analyzed and discussed findings, compared them with existing literature, derived implications for theory and practice, and assessed the predictive validity of the findings to represent the population of ESN users.

\section{Results}

Our study revealed significant aspects relevant to understand the characteristics of different ESN users. As findings, we present (1) a model to affiliate the population of ESN users to archetypical user groups as well as a summary of identified perceived (2) added values and (3) obstacles.

\subsection{Archetypes of ESN users}

As a result of the structuring content analysis [19], we identified six different user types. These different user types were distinguished by the following dimensions: individual openness to ESNs and perceived task-fit (see Figure 1).

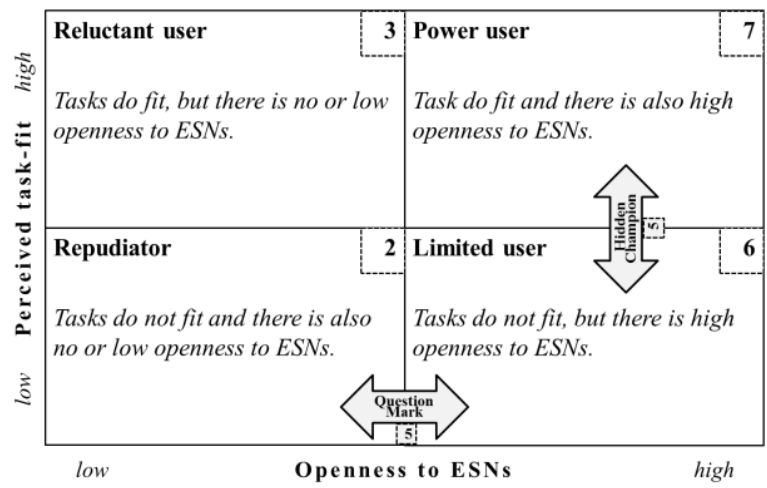

Figure 1. Archetypes of ESN users

In the following, each of the six identified user types are briefly characterized. The characterizations for a power user, a limited user, a reluctant user, and the repudiator are based on the data from seven interviews, six interviews, three interviews, and two interviews, respectively. Each of the two mixed archetypes were deduced from the data of five interviews (as shown in Table 2).

As shown in Table 3, demographics of the interviewees are homogenous for each archetype and do not imply major trends. The data reveals the means of organizational tenure as well as the perceived ESN knowledge and experience of interviewees per archetype. The latter is measured via a 5-point Likert scale (ranging from $1=$ low to $5=$ high) and represents self-perceptions of the interviewees.

4.1.1. Power user. Power users are individuals characterized by a high utilization frequency from several times a day up to a permanent use. They use the ESN actively and as job-related. Therefore, power users appear to strongly contribute to the value adding 
Table 1. Coding categories for archetype characterization

\begin{tabular}{|c|c|c|c|c|}
\hline Dimension & $\begin{array}{l}\text { Coding from } \\
\text { content analysis }\end{array}$ & Description & $\begin{array}{l}\text { Examples } \\
\text { (interview quotes) }\end{array}$ & $\begin{array}{l}\text { Similarities to } \\
\text { constructs }\end{array}$ \\
\hline \multirow{5}{*}{ 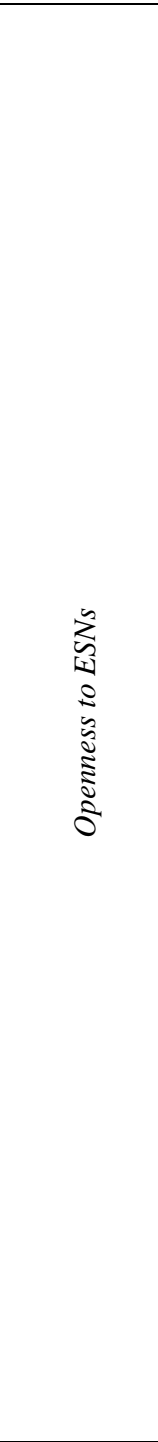 } & $\begin{array}{l}\text { a) Attitude towards } \\
\text { ESNs }\end{array}$ & $\begin{array}{l}\text { Includes individuals' } \\
\text { attitude to the ESN; e.g., } \\
\text { traits as curiosity and } \\
\text { enthusiasm that describe } \\
\text { their individual openness to } \\
\text { the ESN. }\end{array}$ & $\begin{array}{l}\text { High: "I found this very exciting; I was } \\
\text { looking forward to work with a modern } \\
\text { tool like our ESN." [ESNHR06] } \\
\text { Low: "I do not care whether the social } \\
\text { network is available or not. I am } \\
\text { passionless about it." [ ESNLD06] }\end{array}$ & $\begin{array}{l}\text { Computer } \\
\text { Playfulness } \\
\text { [33]/ TAM3, } \\
\text { Perceived } \\
\text { Enjoyment } \\
\text { [31]/TAM3 }\end{array}$ \\
\hline & $\begin{array}{l}\text { b) Perceived ability } \\
\text { to use ESNs }\end{array}$ & $\begin{array}{l}\text { Includes perceived ability } \\
\text { to use the ESN; and also } \\
\text { covers opinions about the } \\
\text { necessity of trainings that } \\
\text { describes their openness to } \\
\text { the ESN. }\end{array}$ & $\begin{array}{l}\text { High: "It is really well done and I find } \\
\text { the handling, once you have used it, not } \\
\text { difficult." [ESNSU05] } \\
\text { Low: "It's a fact that I'm not } \\
\text { accustomed to communicate via the } \\
\text { ESN." [ESNLD03] }\end{array}$ & $\begin{array}{l}\text { Computer } \\
\text { Self-Efficacy } \\
\text { [5]/TAM3, } \\
\text { Complexity } \\
\text { [23]/DOI }\end{array}$ \\
\hline & $\begin{array}{l}\text { c) Promotion of } \\
\text { and resistance } \\
\text { against ESNs }\end{array}$ & $\begin{array}{l}\text { Includes individuals' } \\
\text { attitude and behavior to } \\
\text { promote and support the } \\
\text { ESN within the } \\
\text { organization; it also covers } \\
\text { negative promotion and } \\
\text { resistance against the ESN. }\end{array}$ & $\begin{array}{l}\text { Promotion: "I use it actively and need } \\
\text { those who want to work with me to do } \\
\text { this too. [...] If possible, I use other } \\
\text { tools to direct people towards the social } \\
\text { network." [ESNEN04] } \\
\text { Resistance: "If it were up to me, we } \\
\text { could abolish that." [ESNHR05] }\end{array}$ & $\begin{array}{l}\text { Computer } \\
\text { Anxiety } \\
\text { [31]/TAM3 }\end{array}$ \\
\hline & $\begin{array}{l}\text { d) Perceived future } \\
\text { potential and } \\
\text { importance of } \\
\text { ESNs }\end{array}$ & $\begin{array}{l}\text { Includes individuals' } \\
\text { opinion about the } \\
\text { importance and future } \\
\text { potential of the ESN that } \\
\text { describes their openness to } \\
\text { the ESN. }\end{array}$ & $\begin{array}{l}\text { High: "The future is a globalized world, } \\
\text { we have a lot of knowledge to exchange, } \\
\text { and our network is actually the best way } \\
\text { to filter content quickly. [... I It has a } \\
\text { very high value and it will still } \\
\text { contribute very strongly to how our } \\
\text { company will develop." [ESNHR07] } \\
\text { Low: "Well, if such a network is } \\
\text { properly used, then it could have a } \\
\text { meaning." [ESNEN02] }\end{array}$ & $\begin{array}{l}\text { Long-Term } \\
\text { Consequences } \\
{[26]}\end{array}$ \\
\hline & 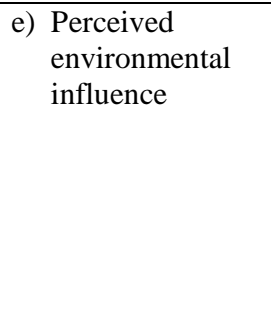 & $\begin{array}{l}\text { Includes perceptions about } \\
\text { the usage behavior of } \\
\text { coworkers regarding the } \\
\text { ESN as well as perceptions } \\
\text { about the environment (e.g. } \\
\text { the organization, } \\
\text { executives) that affect their } \\
\text { individual openness to the } \\
\text { ESN. }\end{array}$ & $\begin{array}{l}\text { High: "My boss communicates mainly } \\
\text { via the social network. Of course, you } \\
\text { are asked to use the social network too." } \\
\text { [ESNSU06] } \\
\text { Low: "But nothing happens: no articles, } \\
\text { no news, no discussions are posted, and } \\
\text { since it is similar seen by colleagues, we } \\
\text { do not use it." [ESNEN02] }\end{array}$ & $\begin{array}{l}\text { Subjective } \\
\text { Norm } \\
\text { [30]/TAM3, } \\
\text { Collective } \\
\text { System Usage } \\
{[4]}\end{array}$ \\
\hline \multirow{2}{*}{ 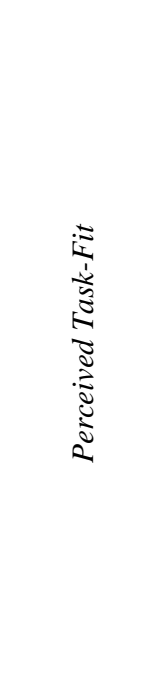 } & $\begin{array}{l}\text { a) Usage type, } \\
\text { frequency and } \\
\text { relative } \\
\text { importance }\end{array}$ & $\begin{array}{l}\text { Includes the type of } \\
\text { business related usage of } \\
\text { the ESN as well as the } \\
\text { frequency; and also covers } \\
\text { their opinion about the } \\
\text { relative importance of the } \\
\text { ESN to assess the } \\
\text { individual fit between job } \\
\text { tasks and ESN. }\end{array}$ & $\begin{array}{l}\text { High: "I try to maximize my efficiency. } \\
\text { For the special thing I do, the social } \\
\text { network is very helpful. Without the } \\
\text { social network, I could not cope with } \\
\text { these volumes in quality and time." } \\
\text { [ESNEN04] } \\
\text { Low: "It's not important for me, I do not } \\
\text { use it." [ESNLD06] }\end{array}$ & $\begin{array}{l}\text { Job Relevance } \\
\text { [30]/TAM3, } \\
\text { Job Fit } \\
{[26]}\end{array}$ \\
\hline & $\begin{array}{l}\text { b) Perceived task \& } \\
\text { outcome related } \\
\text { added value and } \\
\text { obstacles }\end{array}$ & $\begin{array}{l}\text { Includes business related } \\
\text { perceived added values and } \\
\text { obstacles that affect the } \\
\text { individual fit between job } \\
\text { tasks and ESNs. }\end{array}$ & $\begin{array}{l}\text { Added value: "I also use the ESN to put } \\
\text { together collaborative notes. We use it } \\
\text { for brainstorming or to prepare training } \\
\text { materials." [ESNHR04] } \\
\text { Obstacle: "I would like to post all sorts } \\
\text { of mass mails via the social network [...] } \\
\text { unfortunately I cannot, as not all people } \\
\text { use the network and the usage is not } \\
\text { obligatory." [ESNEN05] }\end{array}$ & $\begin{array}{l}\text { Output } \\
\text { Quality [30]/ } \\
\text { TAM3, Task } \\
\text { Requirements } \\
\text { and Tool } \\
\text { Functionality } \\
\text { [11]/TTF } \\
\text { Relative } \\
\text { Advantage } \\
\text { [24]/DOI }\end{array}$ \\
\hline
\end{tabular}




\section{Table 2. Interviewees and their archetype characterization}

\begin{tabular}{|c|c|c|c|c|c|}
\hline Archetype & 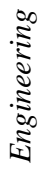 & $\$$ & 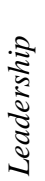 & 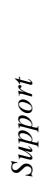 & $\vec{\Xi}$ \\
\hline Power User & 1 & 4 & 1 & 1 & 7 \\
\hline Limited User & 0 & 2 & 2 & 2 & 6 \\
\hline Reluctant User & 1 & 1 & 1 & 0 & 3 \\
\hline Repudiator & 1 & 0 & 1 & 0 & 2 \\
\hline \multicolumn{6}{|l|}{ Mixed Archetype } \\
\hline Hidden Champion & 1 & 1 & 1 & 2 & 5 \\
\hline Question Mark & 1 & 0 & 1 & 3 & 5 \\
\hline
\end{tabular}

Table 3. Demographics by archetypes

\begin{tabular}{|c|c|c|c|c|c|c|c|c|}
\hline & \multicolumn{5}{|c|}{ Age Class } & \multirow{2}{*}{ 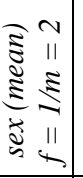 } & \multirow{2}{*}{ 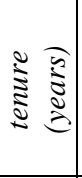 } & \multirow{2}{*}{ 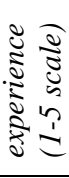 } \\
\hline & 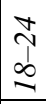 & $\begin{array}{l}\vec{\pi} \\
\tilde{n} \\
\sim \\
\sim\end{array}$ & $\begin{array}{l}7 \\
7 \\
n\end{array}$ & $\begin{array}{l}\pi \\
\frac{\pi}{7} \\
\frac{5}{7}\end{array}$ & $\stackrel{+}{n}$ & & & \\
\hline Power User & 0 & 5 & 0 & 1 & 1 & 1.4 & 12.1 & 4.4 \\
\hline Limited User & 0 & 3 & 2 & 1 & 0 & 1.3 & 5.8 & 3.2 \\
\hline Reluctant User & 0 & 1 & 1 & 0 & 1 & 1.7 & 17.7 & 3.3 \\
\hline Repudiator & 0 & 0 & 1 & 1 & 0 & 1.5 & 9.5 & 3.5 \\
\hline Hidden Champion & 3 & 0 & 1 & 1 & 0 & 1.8 & 6.6 & 3.4 \\
\hline Question Mark & 1 & 1 & 1 & 1 & 1 & 1.4 & 13.4 & 2.4 \\
\hline Total & 4 & 10 & 6 & 5 & 3 & 1.5 & 10.4 & 3.4 \\
\hline
\end{tabular}

content generation in the social network of an organization. This user type is extremely open to the decreasing importance of hierarchy and distance within an organization as experienced with the introduction of an ESN. Pronounced IT-competence of the power user paired with high curiosity and anticipation emphasizes the enthusiasm to attempt new things. Individuals of this type are very familiar with using the social network and fully capable of taking advantage of the same, such as performing tasks for daily business with more efficiency, to reach a wider range of colleagues or to support team work and global cooperation. Power users pursue the approach to work mainly via the social network. Power users highly perceive the creation of values when performing business task. Power users focus on high utilization intensity, and therefore analyze their environment and attempt to inspire colleagues through high levels of promotion and support of the system. According to power users, the system significantly contributes to the success of the company in the future, and thus the ESN is considered as highly important by them.

4.1.2. Repudiator. A repudiator is extremely opposite to a power user. This is significantly reflected in the low utilization frequency of the ESN, which includes mainly a passive usage from only once or twice a month to gather information of a general type. Although repudiators state that they are open to new things, the ESN must first be obviously connected with added values for them. This is not the case and repudiators do not see any added value with the system, and thus their openness to the ESN and subsequently their intention to use the system is low. The social network is not seen as an aid. According to repudiators, the communication for their type of work is better covered by other existing tools in the firm such as email or instant messaging. Additionally, the reversal of the push to pull mechanism for gathering information is rejected by individuals of this type and they refuse to pull information from the executives. Conversely, they prefer that information is sent to them. A mandatory use of the system is rejected by repudiators and is underlined by resistance. The ESN is considered by them as unnecessary and not important. Thus, they predict only a low future potential for the ESN.

4.1.3. Limited user. Limited users have an open attitude to the introduction and usage of the ESN within the organization. Their utilization frequency of the system from a weekly to a daily basis is mainly passive to gather general information, which is not related to a specific task. Despite the low task-fit, limited users have high levels of intention toward a more task-related usage of the system. In an exemplary manner for a low task-fit, limited users mentioned tasks that include work with personal or security sensitive data. Due to the low levels of support for these users' tasks and their daily business, the ESN is only slightly important for them. Despite perceived obstacles of a low task-fit, limited users are always interested in increased utilization intensity to expand their own usage of the ESN. This underlines their intention to push the usage of the system to a higher level. Limited users consider ESN as highly important for the future as well as decisive for the future success of the company.

4.1.4. Reluctant user. Reluctant users have a medium utilization frequency of the ESN from a weekly to a daily basis. This usage is slightly more passive than active although it is supported by a high task-fit and consequently contributes to a high support level for business related tasks such as using the system for document collaboration or as a knowledge database. Reluctant users perceive task-fit as high, and thus equal to that perceived by power users. Reluctant users like the idea of introducing the ESN within the organization although they do not perceive an increase in efficiency 
while using it. According to them, the system is not user friendly, and it is not a tool, that is recognized as well-engineered or fully utilized. Reluctant users are used to working with email to perform business tasks. Individuals of this user type exhibit resistance against the ESN, and therefore they try to circumvent its use. According to them, results are achieved better and faster with other established tools within the organization. Additionally, the ESN is observed to contribute to information overload. Although they use the system, reluctant users perceive added values only to a very low extent. They appreciate the system to procure general and not task-related information or for private exchange. Reluctant users characterize ESN usage as leading to duplication and a decrease in productivity. Reluctant users work around ESN despite a high task-fit. They use the system only when they are forced to such as if other colleagues indirectly push them to do so. Reluctant users reject the mandatory or exclusive use of the ESN. Interestingly, reluctant users believe that an ESN maximizes the success of a company although it will not be the decisive and only medium for an organization's communication.

4.1.5. Hidden champion. In addition to the predefined four user types in this paper, two further mixed user types were identified. A hidden champion is characterized by a high openness to ESNs and a medium task-fit level. Thus, hidden champions are classified halfway between limited and power users.

4.1.6. Question mark. The second mixed user type, namely a question mark, is characterized by a medium openness to ESNs and a low task-fit level. Thus, Question Marks are classified halfway between repudiators and limited users.

\subsection{Perceived values by archetypes}

The overall results are strongly in line with previous study. Table 4 presents an overview. Here, improved communication across hierarchical and organizational boundaries [7], [36], improved knowledge transfer and expert search [2], [9], [14], [17], enhanced innovational strength [11], [14], [18] as well as the establishment and strengthening of social ties [12], [27] were confirmed as benefits of ESN.

The communication via ESN is more direct, open, and transparent. Information and content are shared easily. ESN users decide whenever they want to read the latest information from the network, and this appeared to lead to a reduction in emails and, interestingly to shorter content. Members can spread information to large communities and even to the entire ESN. Table 4. Perceived added values by
archetypes

\begin{tabular}{|l|l|l|}
\hline Archetype & Perceived Added Value & $n$ \\
\hline $\begin{array}{l}\text { Power } \\
\text { User }\end{array}$ & $\begin{array}{l}- \text { high business-related task support } \\
\text { enabler for new and more efficient } \\
\text { methods of working together }\end{array}$ & 7 \\
increased efficiency (substitution of \\
prevalent tools) & 5 \\
- $\begin{array}{l}\text { imhanced meta knowledge and } \\
\text { contribution to corporate success } \\
\text { creation of innovational strength }\end{array}$ & 5 \\
\hline $\begin{array}{l}\text { Limited } \\
\text { User }\end{array}$ & $\begin{array}{l}\text { - enhanced information handling, e.g. } \\
\text { addressing communities or gathering } \\
\text { company related information } \\
\text { information and experts is obtained be } \\
\text { found faster via ESNs }\end{array}$ & 2 \\
\hline $\begin{array}{l}\text { Reluctant } \\
\text { User }\end{array}$ & $\begin{array}{l}\text { useful to derive general information } \\
\text { outside business related tasks }\end{array}$ & 2 \\
\hline Repudiator & $-\quad$ ESNs provide no added value & 2 \\
\hline
\end{tabular}

Colleagues are represented via personal profiles, and they are visible as authors and available for questions from other members. This fosters new relationships and strengthens social ties. Additionally, the ESN serves as a central and continual knowledge base with the ability to access or edit content at any time. This avoids duplication where users redundantly save data and information in their mailboxes such as while using email. The ESN information can be posted and collected thematically within communities based on the interests of their members.

The network is also used for documentation such as users coordinating and communicating content. This avoids work duplication and may help in increasing employee performance. The ESN provides an easy way for its members to ask questions to a large target audience, without even knowing a single colleague or expert. Conversely, members may also purposefully pose their questions within communities to deliberately restrict the circle of colleagues such as when their question matches the field of a certain community.

Hence, for questions to a broad audience, the enquirers may get a faster response from other ESN members than they may expect when compared to email. Furthermore, simultaneous topics for group discussions may be created when individuals ask questions within communities. The search for colleagues or experts may be accelerated via the ESN since connections between members and their content are transparent and visible for third parties [14]. This is further facilitated by the fact that individuals represent 
their interests and activities via a personal profile. Contacts and friendships also foster idea generation within communities and between colleagues. Thus, the innovative power of any organization is enhanced. Ideas can be generated in real time or simultaneously such as for brainstorming colleagues working together on a collaborative note. Furthermore, a few respondents believe that the ESN creates an additional competitive advantage such as prospective new employees that prefer to work for an organization with this modern communication tool.

With respect to power users and limited users, the ESN improves communication and information sharing, and therefore allows a more efficient way to work together. In contrast, reluctant users only perceive added values in the procurement of general information outside their business such as sharing news about the firm or exchanging non-job-related content. Ultimately, repudiators do not see any value contribution through an ESN.

\subsection{Perceived obstacles by archetypes}

Power users mainly criticize that the cultural change has not occurred as yet within an organization. According to them, voluntary use prevents a higher generation of value across the whole enterprise. Specifically, executives must increasingly use the system in a productive way that includes a bidirectional communication and collaboration with employees. Power users are likely to reach more colleagues via the ESN. They believe that an ESN should be the exclusive tool for certain operations within the organization. Conversely, repudiators complain that the ESN doesn't support their daily business tasks and that it lacks basic requirements. An even worse implication is that they perceive ESN use to lead to work duplication and work overhead.

A serious stated problem is that the responsibility is passed from leadership level to employees. According to repudiators, the ESN is not a solution for better communication within the organization. Limited users are restricted in their usage of ESNs due to a broader range of obstacles as follows. First, they believe that the ESN is not yet fully accepted within the organization. This and voluntary use impede increased utilization of the system. In order to reach colleagues, the use of email is necessary for limited users. This results in work duplication, which impedes the increased utilization of the system. According to limited users, the network and communities should be more restrictive such as prioritizing business activities. Limited users complain about the excessive amount of tools in use, i.e., the ESN should be the only archive for any type of information. In contrast, they believe that internal communications cannot be limited only to the network such as the sharing of sensitive data or working on security-related projects. A reason is that encrypted data cannot be transmitted via the ESN. Lack of trust in the system further contributes to limited usage such as the absence of assurance in individuals about the circle of recipients of a message and that recipients read a message on time. Therefore, the system is not very suitable for direct communication. Additionally, the ESN is designated for internal communication, and thus it is not appropriate for communication and work with clients. Reluctant users believe that the system continues to contribute to information overload. According to them, it is difficult to determine important or relevant information via the ESN. Their usage of the ESN leads to work duplication and work overhead. The system is confusing to them and this results in a high amount of information albeit with poor quality. Furthermore, they are concerned about the absence of uniform rules about how to use the ESN and they remain uncertain about the circle of recipients of a message and that answers do not appear in a timely manner. Moreover, the tagging of the importance of information is omitted as reluctant users expect it from other systems such as

\section{Table 5. Perceived obstacles by archetypes}

\begin{tabular}{|c|c|c|}
\hline Archetype & Perceived Obstacles & $n$ \\
\hline Power User & 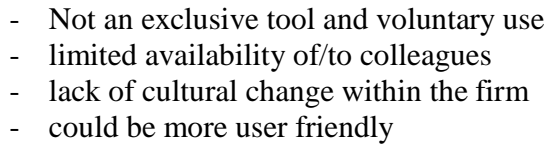 & $\begin{array}{l}6 \\
5 \\
3 \\
2\end{array}$ \\
\hline $\begin{array}{l}\text { Limited } \\
\text { User }\end{array}$ & $\begin{array}{ll}\text { - } & \text { no exclusive tool, voluntary and } \\
& \text { unrestricted usage } \\
\text { - } & \text { limited business-related task support } \\
\text { - } & \text { lack of acceptance within the firm } \\
\text { - } & \text { lack of trust and uncertainty in usage } \\
\text { - } & \text { not applicable for sensitive data } \\
\text { - } & \text { lack of knowledge in using the system } \\
\text { - } & \text { leads to work duplication }\end{array}$ & $\begin{array}{l}6 \\
4 \\
4 \\
3 \\
3 \\
2 \\
2\end{array}$ \\
\hline $\begin{array}{l}\text { Reluctant } \\
\text { User }\end{array}$ & $\begin{array}{ll}\text { - } & \text { lack of acceptance within the firm } \\
\text { - } & \text { leads to information overload } \\
\text { - } & \text { leads to work duplication/overhead } \\
\text { - } & \text { difficult and confusing usage } \\
\text { - } & \text { lack of trust and uncertainty in usage } \\
\text { - } & \text { voluntary and unrestricted usage }\end{array}$ & $\begin{array}{l}3 \\
3 \\
2 \\
2 \\
2 \\
1\end{array}$ \\
\hline Repudiator & $\begin{array}{ll}\text { - } & \text { low/no business-related task support } \\
& \text { and no perceived added value } \\
\text { - } & \text { unclear responsibilities } \\
\text { - } & \text { personal work load situation } \\
\text { - } & \text { leads to work duplication/overhead } \\
\text { - } & \text { people important to them reject ESNs } \\
\text { - } & \text { lacks basic requirements to be useful } \\
\text { - } & \text { leads to information overload }\end{array}$ & $\begin{array}{l}2 \\
2 \\
2 \\
2 \\
1 \\
1\end{array}$ \\
\hline
\end{tabular}


filtering the importance of information via a sender of an email. In a manner similar to limited users, reluctant users complain that the ESN is not yet fully accepted within the company. An overview of the perceived obstacles is listed in Table 5.

\section{Discussion}

The case study shows that different archetypes of ESN users exist. Furthermore, we reveal that ESN users are characterized via the introduced model by the following dimensions: openness to ESN and perceived task-fit.

\subsection{Implications for theory and practice}

An individual perception of "what is in it for me?" appears to be reflected in the constructs that were coded. This supports the crafted dimensions as valid and purposeful. Specifically, users already experienced the ESN, and thus it appears logical and feasible that the critical drivers of ESN use can be condensed via the sense-making and work-productivity-rising constructs that were deduced from the interview data. Therefore, in contrast to recent studies by Leonardi [14], we added an axis "perceived task-fit" that captures the importance and relevance of task-fit.

The dimensions and constructs coded show similarities with the constructs in existing literature (see Table 1). This seems understandable as we consulted existing user acceptance models to prepare the interview guide. However, none of the existing studies include the model components as a whole. This might be attributed to the novelty of the approach to investigate the archetypes of ESN users.

In contrast to age, sex, tenure, and ESN experience, only the participants' business role predicts the identified archetypes. While it is understandable that human resources and business support indicates a generation of higher task-fit and openness to ESN scores given that their jobs require good communication skills, surprisingly, leaders who actually introduced the ESN do not. This might refer to the explicit request for open feedback about their personal experience with ESN use. Similar to leaders, engineers do not indicate a trend towards certain archetypes within the model. This might be related to the idea that engineers are not perceived as "people persons" but perceived as interested in technical solutions. These two influences may balance the results such that they do not show a noticeable trend. An interesting next step for a future study could include a better understanding of the impact of business role on the identified archetypes. In summary, both diversity in the sample and the argumentative stable results strongly support the generalizability of the described archetypes within the introduced model.

The introduced model centers on individual perceptions and tendencies of such users that are typical for large industrial organizations, and thus the presented archetypes strongly indicate that they are also generalizable to other ESNs in other organizations.

Furthermore, it is remarkable that identified archetypes show similarities in characteristics with the vocabulary indicated by Rogers [23] who distinguished the adoption of new technology by users as follows: power user and limited user vs. innovator, early adopter and early majority, reluctant user vs. late majority as well as repudiator vs. laggards. In contrast to Rogers [23], this study does not reflect the chronological aspect as this case study was conducted after the ESN was introduced twenty-two months ago.

Except for an obvious link in their dominant usage type (active/passive), characteristics of archetypes do not indicate a link to certain roles in any kind of community of practice as described in literature [28], [13]. However, the general perspective that it is not feasible to expect that all individuals should use a system with equal frequency is reflected by openness to ESNs and actual use of ESNs scores where both differ significantly. Furthermore, characteristics of archetypes did not indicate a link to a conflict of interest and incentives of ESN users [21]. The rather passive usage of ESN users indicates accordance with the premise that a small minority does most of the work in social networks and online communities [22], [20].

An unanticipated finding was that visual analysis depicted a noticeable "development path" for the identified archetypes beginning with the question mark via limited user and hidden champion to a power user. The "development path" could be used by organizations to actively manage an ESN within the IT landscape in a geared and purposeful manner. This would allow strategic management to develop both employees and an organization individually according to the desired ESN strategy and according to the characteristics of different archetypes. For example, in order to increase usage, power users could be selected and appointed as "ambassadors"; motivational speeches could be given to "question marks"; and business processes could be analyzed and transformed to stronger ingrain ESNs in the IT landscape and thereby address limited users and hidden champions. Therefore, one could infer that the model also allows assessing the staff of a firm according to their appropriateness with respect to their level, efforts 
towards development, and their potential to contribute in firms of the future.

\subsection{Threats for validity}

Subsequently, we discuss possible threats for the validity of the purely exploratory research design (comprised of a single case study with $n=28$ ) to understand the value of the presented results.

First, although, common sense indicates that employees in the IT domain would be rather open to social software, the sample appears carefully selected and reasonable to represent an industry independent organizational setup that support results that are not deviated by potential specifics of an IT company. In contrast to prior research that focuses on single business units [14], the aim of the study involves investigating ESN users on a general company level, a future study with a sample comprised of more companies could help in strengthening the validity of the presented results.

Second, the last three interviews do not reveal new aspects, and thus the sample size appears reasonable and adequate for the exploratory approach.

Third, the comparison of (a) recognized definitions of ESNs with (b) the actual ESN key functionalities at the case company (see introduction section) strongly indicate that the results are not deviated by the specifics of the actual ESN design.

Fourth, a further potential limitation of the study is that findings are obtained from the perceptions and opinions of the subjects and may be influenced by a "social desirability bias". We carefully crafted our interview guide to avoid the same, such as frequently asked for examples, and moved to fictitious situations whenever we felt that interviewees were uncomfortable with the situation [19].

\section{Conclusion}

The aim of the study involves increasing an understanding of the usage of ESNs within large organizations. In order to examine whether members of an ESN are divided into different user types, we posed the following research questions: "what are the archetypical users of ESN systems," "how users value the ESN system they use," and "what kind of obstacles they perceive".

Although, previous studies were relevant for user typification, there is a paucity of studies proposing an approach that combines individual and productivity related dimensions. This study presents a model to (a) characterize ESN users and (b) classify them as archetypes based on the following two dimensions: openness to ESNs and perceived task-fit.

The results indicated four archetypes of ESN users, namely power users, limited users, reluctant users, and repudiators and two mixed user types, namely hidden champions and question marks. Additionally, the results show that users perceive added values and obstacles of ESNs differently.

From a practical viewpoint, the model contributes to the challenges for the organizational development of large organizations that intend to increase ESN use. A future study will comprise of field experiments that use the introduced approach of characterizing ESN users in a large business unit and develop and apply measures to address different archetypes based on their characteristics to improve ESN utilization intensity.

From a theoretical viewpoint, the study contributes to the discussion on user typification, user acceptance, utilization intensity, and value contribution of ESNs in large organizations.

\section{References}

[1] boyd, d.m. and N.B. Ellison, "Social Network Sites: Definition, History, and Scholarship", Journal of ComputerMediated Communication, 13(1), 2007, pp. 210-230.

[2] Bughin, J., "The rise of enterprise 2.0", Journal of Direct, Data and Digital Marketing Practice, 9(3), 2008, pp. 251259.

[3] Burghin, J., "Look Beyond Your "Social Media Presence": https://hbr.org/2013/01/look-beyond-a-sociamedia-presence", 2013.

[4] Burton-Jones, A. and G.S. Hubona, "Individual differences and usage behavior: Revisiting a technology acceptance model assumption", ACM Sigmis Database, 36(2), 2005, pp. 58-77.

[5] Compeau, D.R. and C.A. Higgins, "Computer selfefficacy: Development of a measure and initial test", MIS quarterly, 1995, pp. 189-211.

[6] Davis, F.D., "Perceived usefulness, perceived ease of use, and user acceptance of information technology", MIS quarterly, 1989, pp. 319-340.

[7] DiMicco, J., D.R. Millen, W. Geyer, C. Dugan, B. Brownholtz, and M. Muller, "Motivations for social networking at work", in Proceedings of the 2008 ACM conference on Computer supported cooperative work, B. Begole, Editor, the ACM 2008 conference, San Diego, CA, USA, 08.11.2008 - 12.11.2008. 2008. ACM: New York, NY.

[8] Ellison, N.B., J.L. Gibbs, and M.S. Weber, "The Use of Enterprise Social Network Sites for Knowledge Sharing in Distributed Organizations", American Behavioral Scientist, 59(1), 2015, pp. 103-123. 
[9] Fulk, J. and Y.C. Yuan, "Location, Motivation, and Social Capitalization via Enterprise Social Networking", Journal of Computer-Mediated Communication, 19(1), 2013, pp. 20-37.

[10] Goodhue, D.L. and R.L. Thompson, "Task-technology fit and individual performance", MIS quarterly, 1995, pp. 213-236.

[11] Hasan, H. and C. Pfaff, "The Wiki: an environment to revolutionise employees' interaction with corporate knowledge", International Conference on Advances in Computer-Human Interactions, 2006, pp. 377-380.

[12] Jackson, A., J. Yates, and W. Orlikowski, "Corporate Blogging: Building community through persistent digital talk", in 40th Annual Hawaii International Conference on System Sciences, 40th Annual Hawaii International Conference on System Sciences. 2007.

[13] Jahnke, I., "Dynamics of social roles in a knowledge management community", Computers in Human Behavior, 26(4), 2010, pp. 533-546.

[14] Leonardi, P.M., "Social Media, Knowledge Sharing, and Innovation: Toward a Theory of Communication Visibility", Information Systems Research, 25(4), 2014, pp. 796-816.

[15] Leonardi, P.M., M. Huysman, and C. Steinfield, "Enterprise social media: Definition, history, and prospects for the study of social technologies in organizations", Journal of Computer-Mediated Communication, 19(1), 2013, pp. 119.

[16] Mann, J., T. Austin, N. Drakos, C. Rozwell, and A. Walls, "Predicts 2013: Social and Collaboration Go Deeper and Wider", Gartner Inc. report, 2012.

[17] Mansour, O., M. Abusalah, and L. Askenäs, "Wiki collaboration in organizaitons: An exploratory study", In proceedings of European Computer Information Systems Conference Paper 265, 2011.

[18] Mäntymäki, M. and K. Riemer, eds., Information, ideas and input: The value of enterprise social networks, ACIS, 2014.

[19] Miles, M.B. and A.M. Huberman, Qualitative data analysis: An expanded sourcebook, sage, 1994.

[20] Okoli, C., M. Mehdi, M. Mesgari, F.Å. Nielsen, and A. Lanamäki, "Wikipedia in the eyes of its beholders: A systematic review of scholarly research on Wikipedia readers and readership", Journal of the Association for Information Science and Technology, 65(12), 2014, pp. 2381-2403.

[21] Orlikowski, W.J., "Learning from notes: Organizational issues in groupware implementation", in Proceedings of the 1992 ACM conference on Computer-supported cooperative work. 1992.
[22] Preece, J. and B. Shneiderman, "The reader-to-leader framework: Motivating technology-mediated social participation", AIS Transactions on Human-Computer Interaction, 1(1), 2009, pp. 13-32.

[23] Rogers, E.M., "Elements of diffusion", Diffusion of innovations, 5, 2003, pp. 1-38.

[24] Rossmann, A. and G. Stei, "Enterprise Social NetworksEinführung in die Thematik und Ableitung relevanter Forschungsfelder", in Enterprise Social Networks. 2016. Springer.

[25] Samaradiwakara, G. and C.G. Gunawardena, "Comparison of existing technology acceptance theories and models to suggest a well improved theory/model", International Technical Sciences Journal, 2014, pp. 21-36.

[26] Thompson, R.L., C.A. Higgins, and J.M. Howell, "Personal computing: Toward a conceptual model of utilization", MIS quarterly, 1991, pp. 125-143.

[27] Thom-Santelli, J., M.J. Muller, and D.R. Millen, "Social tagging roles: Publishers, evangelists, leaders", in Proceedings of the SIGCHI Conference on Human Factors in Computing Systems. 2008.

[28] Velasquez, A., R. Wash, C. Lampe, and T. Bjornrud, "Latent users in an online user-generated content community", Computer Supported Cooperative Work (CSCW), 23(1), 2014, pp. 21-50.

[29] Venkatesh, V. and H. Bala, "Technology acceptance model 3 and a research agenda on interventions", Decision sciences, 39(2), 2008, pp. 273-315.

[30] Venkatesh, V. and F.D. Davis, "A theoretical extension of the technology acceptance model: Four longitudinal field studies", Management science, 46(2), 2000, pp. 186-204.

[31] Venkatesh, V. and M.G. Morris, "Why don't men ever stop to ask for directions? Gender, social influence, and their role in technology acceptance and usage behavior", MIS quarterly, 2000, pp. 115-139.

[32] Venkatesh, V., M.G. Morris, G.B. Davis, and F.D. Davis, "User acceptance of information technology: Toward a unified view", MIS quarterly, 2003, pp. 425-478.

[33] Webster, J. and J.J. Martocchio, "Microcomputer playfulness: Development of a measure with workplace implications", MIS quarterly, 1992, pp. 201-226.

[34] Williams, S.P., V. Hausmann, C.A. Hardy, and P. Schubert, "Enterprise 2.0 Research: Meeting the challenges of practice", BLED 2013 Proc, 2013, pp. 251-26.

[35] Yin, R.K., Case study research: Design and methods, 5th edn., SAGE Publ, Los Angeles CA u.a., 2014.

[36] Zhang, J., Y. Qu, J. Cody, and Y. Wu, "A Case Study of Micro-blogging in the Enterprise: Use, Value, and Related Issues", Proceedings of the SIGCHI Conference on Human Factors in Computing Systems, 2010, pp. 123-132. 